\title{
Article \\ Discovery of GOT1 Inhibitors from a Marine-Derived Aspergillus terreus That Act against Pancreatic Ductal Adenocarcinoma
}

\author{
Shan Yan ${ }^{1,+}$, Changxing $\mathrm{Qi}^{1,+}{ }^{\dagger}$, Wei Song ${ }^{2}$, Qianqian $\mathrm{Xu}{ }^{1}$, Lianghu Gu ${ }^{1}$, Weiguang Sun ${ }^{1, *}$ \\ and Yonghui Zhang $1, * \mathbb{D}$
}

1 Hubei Key Laboratory of Natural Medicinal Chemistry and Resource Evaluation, School of Pharmacy, Tongii Medical College, Huazhong University of Science and Technology, Wuhan 430030, China; yanshanwhu@163.com (S.Y.); qichangxing@hust.edu.cn (C.Q.); Xuxuqq@hust.edu.cn (Q.X.); gulianghu@hust.edu.cn (L.G.)

2 Guangdong Provincial Key Laboratory of Microbial Culture Collection and Application, State Key Laboratory of Applied Microbiology Southern China, Institute of Microbiology, Guangdong Academy of Sciences, Guangzhou 510070, China; 20191103@zcmu.edu.cn

* Correspondence: weiguang_sun@hust.edu.cn (W.S.); zhangyh@mails.tjmu.edu.cn (Y.Z.)

+ S.Y. and C.Q. contributed equally to this work.

check for updates

Citation: Yan, S.; Qi, C.; Song, W.; Xu, Q.; Gu, L.; Sun, W.; Zhang, Y. Discovery of GOT1 Inhibitors from a Marine-Derived Aspergillus terreus That Act against Pancreatic Ductal Adenocarcinoma. Mar. Drugs 2021, 19, 588. https://doi.org/10.3390/ md19110588

Academic Editor:

Ekaterina Yurchenko

Received: 26 September 2021

Accepted: 18 October 2021

Published: 20 October 2021

Publisher's Note: MDPI stays neutral with regard to jurisdictional claims in published maps and institutional affiliations.

Copyright: (c) 2021 by the authors. Licensee MDPI, Basel, Switzerland. This article is an open access article distributed under the terms and conditions of the Creative Commons Attribution (CC BY) license (https:/ / creativecommons.org/licenses/by/ $4.0 /)$.

\begin{abstract}
Pancreatic ductal adenocarcinoma (PDAC) is a devastating digestive system carcinoma with high incidence and death rates. PDAC cells are dependent on the Gln metabolism, which can preferentially utilize glutamic oxaloacetate transaminase 1 (GOT1) to maintain the redox homeostasis of cancer cells. Therefore, small molecule inhibitors targeting GOT1 can be used as a new strategy for developing cancer therapies. In this study, 18 butyrolactone derivatives (1-18) were isolated from a marine-derived Aspergillus terreus, and asperteretone B (5), aspulvinone $\mathrm{H}(\mathrm{AH}, 6)$, and (+)$3^{\prime}, 3^{\prime}$-di-(dimethylallyl)-butyrolactone II (12) were discovered to possess significant GOT1-inhibitory activities in vitro, with $\mathrm{IC}_{50}$ values of $(19.16 \pm 0.15),(5.91 \pm 0.04)$, and $(26.38 \pm 0.1) \mu \mathrm{M}$, respectively. Significantly, the molecular mechanism of the crystal structure of GOT1-AH was elucidated, wherein $\mathrm{AH}$ and the cofactor pyrido-aldehyde 5-phosphate competitively bound to the active sites of GOT1. More importantly, although the crystal structure of GOT1 has been discovered, the complex structure of GOT1 and its inhibitors has never been obtained, and the crystal structure of GOT1-AH is the first reported complex structure of GOT1/inhibitor. Further in vitro biological study indicated that AH could suppress glutamine metabolism, making PDAC cells sensitive to oxidative stress and inhibiting cell proliferation. More significantly, AH exhibited potent in vivo antitumor activity in an SW1990-cell-induced xenograft model. These findings suggest that AH could be considered as a promising lead molecule for the development of anti-PDAC agents.
\end{abstract}

Keywords: natural products; GOT1 enzyme; X-ray crystallography; pancreatic ductal adenocarcinoma cells; glutamine metabolism

\section{Introduction}

Pancreatic ductal adenocarcinoma (PDAC), a highly aggressive malignancy, is predicted to become the second leading cause of cancer-related deaths by 2030, despite the fact that 5-year survival rates have consistently remained below 10\% throughout the past several decades [1]. Alteration of metabolism is a key indicator for cancer cells to meet their biosynthesis requirements [2,3]. Recently, it has been demonstrated that the demand for glutamine (Gln) in PDAC cells is excessive, promoting proliferation and tumor growth through a non-canonical Gln metabolic pathway regulated by KRAS [4-6]. In detail, the Gln-derived aspartate (Asp) is transported to the cytoplasm, where glutamicoxaloacetic transaminase 1 (GOT1) catalyzes the transformation of $\alpha$-KG and Asp to Glu and oxaloacetate (OAA), respectively. Subsequently, continuous catalytic reactions of 
malate dehydrogenase 1 (MDH1) and malic enzyme 1 (ME1) convert OAA to pyruvate and yield NADPH (Figure 1A) $[7,8]$. PDAC cells rely on these responses to increase the $\mathrm{NADPH} / \mathrm{NADP}^{+}$ratio in order to maintain the intracellular redox state $[9,10]$. As an essential enzyme in this pathway, the inhibition of GOT1 activity is growth-inhibitory in PDAC cells, but well tolerated by normal cells [11]. GOT1 metabolic pathways have also been shown to play a role in other malignant tumor cells, including small-cell lung cancer, breast adenocarcinoma, and glioblastoma multiforme [12,13]. Hence, GOT1 inhibitors represent a promising tool for studying the targeting of the metabolism of PDAC and other Gln-dependent cancers. In addition, although the crystal structure of GOT1 is known, the complex structure of GOT1 and its inhibitors has never been obtained [14]. Therefore, starting with higher activity GOT1 inhibitors, and combining the study of the crystal structure of GOT1 inhibitors with pharmacological evaluation, is an important approach to developing anti-PDAC drugs [15].
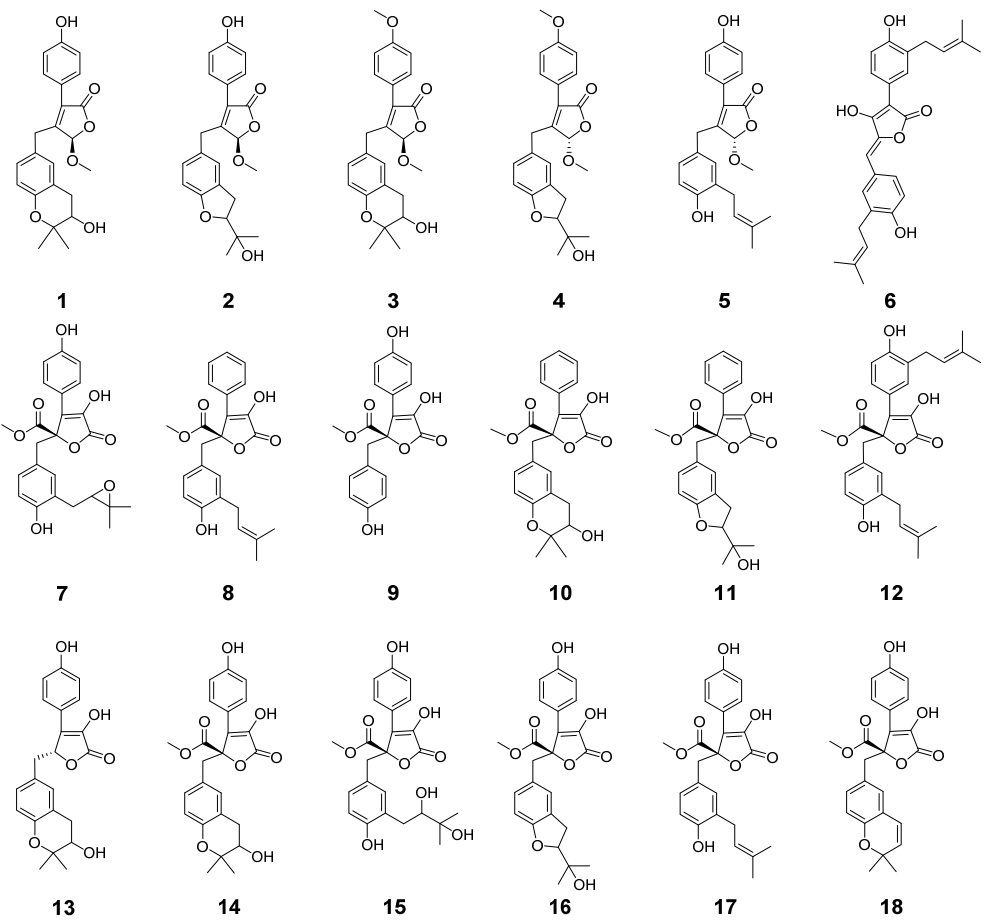

Figure 1. Structures of 1-18.

Butyrolactones, a class of highly functional lignan derivatives, are known as luciferase inhibitors [16]. In our previous study, we identified a potent butyrolactone-type GOT1 inhibitor from soil-sourced Aspergillus terreus collected from the bottom of the Yangzi River, which could suppress PADC cells' growth by interfering with glutamine metabolism [10]. In our continuing study of GOT1-inhibitory metabolites, the chemical constituents of a marinesediment-derived $A$. terreus obtained from the South China Sea were investigated systematically, and 18 butyrolactone derivatives (1-18) were isolated from this fungus (Figure S1). The GOT1-inhibitory activity screening of $\mathbf{1}-\mathbf{1 8}$ suggested that (+)-asperteretone $\mathrm{B}(\mathbf{5})$, aspulvinone $\mathrm{H}(\mathrm{AH}, 6)$, and (+)-3', $3^{\prime}$-di-(dimethylallyl)-butyrolactone II (12) exhibit outstanding GOT1-inhibitory activities in vitro, with IC $_{50}$ values of $(19.16 \pm 0.15),(5.91 \pm 0.04)$, and $(26.38 \pm 0.1) \mu \mathrm{M}$, respectively. Further GOT1-inhibitory assays of AH were performed, and the high-resolution crystal structure of the complex was also obtained, which revealed that AH is a pyridoxal 5-phosphate (PLP) competitor that binds to the active site of GOT1. Simultaneously, we found that AH could inhibit the growth of PDAC cells both in vivo and in vitro, and induce the apoptosis of cancer cells by inhibiting Gln metabolism. As a selective GOT1 inhibitor, the complex crystal structure of GOT1-AH and the biological activity evaluation of $\mathrm{AH}$ will shed new light on the design and development of agents for 
the treatment of PDAC. In this paper, we report the isolation of $\mathbf{1 - 1 8}$, and the co-crystal structure of GOT1-AH, as well as the anti-PDAC biological evaluation of AH.

\section{Results and Discussion}

\subsection{Structural Elucidation}

On the basis of the optical values of $\mathbf{1 - 1 8}$, they were not racemate; corresponding to the spectroscopic characterization-including the values of chemical shifts and other physical constants from the reported papers [17-23] — compounds 1-18 were identified as terrusnolide B (1), terrusnolide D (2), terrusnolide C (3), (+)-asperteretone C (4), (+)-asperteretone B (5), aspulvinone H (6), butyrolactone III (7), versicolactone B (8), butyrolactone II (9), asperlide A (10), asperlide B (11), (+)-3' $3^{\prime}$-di-(dimethylallyl)-butyrolactone II (12), isobutyrolactone V (13), butyrolactone V (14), butyrolactone VI (15), butyrolactone IV (16), butyrolactone I (17), aspernolide E (18) (Figure 1).

\subsection{The GOT1-Inhibitory Activity of 1-18}

Based on our previous study, all of the isolates 1-18 were evaluated for their GOT1inhibitory activity. Among the evaluated isolates, compound 6 (AH) exhibited the best inhibitory effect of GOT1 activity, with an $\mathrm{IC}_{50}$ value of $(6.91 \pm 0.04) \mu \mathrm{M}$; compounds 5 and 12 also exhibited GOT1-inhibitory effects, with $\mathrm{IC}_{50}$ values of $(19.16 \pm 0.15)$ and $(26.38 \pm 0.12) \mu \mathrm{M}$, respectively, while the other isolates showed no obvious activity up to $40 \mu \mathrm{M}$ (Figure 2A-C). Microscale thermophoresis (MST) experiments further confirmed that $\mathrm{AH}$ can bind GOT1, with a $K_{d}$ value of $(2.14 \pm 0.47) \mu \mathrm{M}$ (Figure 2D). These findings suggest that $\mathrm{AH}$ could be used as a lead compound for the design and development of GOT1 inhibitors. Structure-activity relationship analysis showed that the butyrolactonetype natural products possessing the 5-benzylidene-3-phenylfuran-2(5H)-one core might bear considerably stronger GOT1-inhibitory activity, and that the isopentenyl side chains in butyrolactones might also increase the inhibition of GOT1 activity.

A

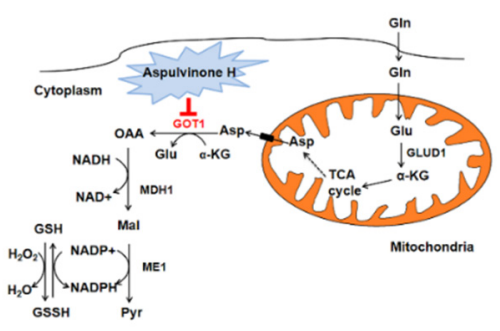

C

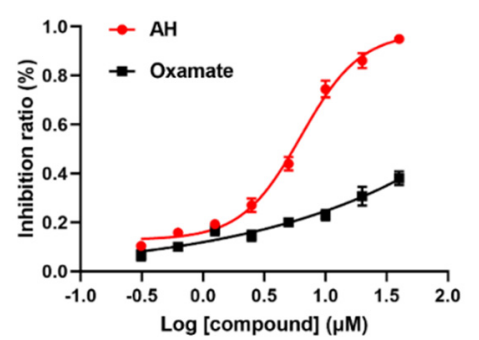

B

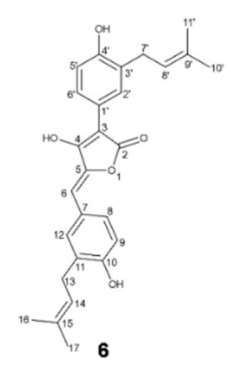

D

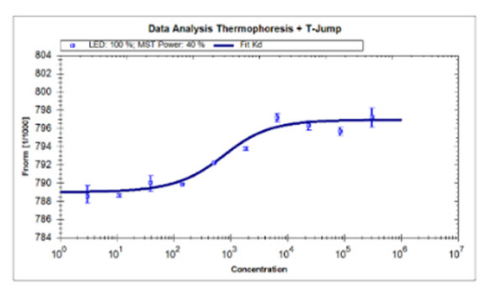

Figure 2. AH selectively targets GOT1: (A) Schematic overview of GOT1-mediated Gln metabolism. (B) The chemical structure of AH. (C) AH selectively inhibits GOT1 activity. (D) The $K_{d}$ value between $\mathrm{AH}$ and GOT1, as detected via MST assay.

\subsection{Co-Crystal Structure of GOT1 and AH}

The crystal structure of GOT1 has been known for many years, but the co-crystal structure of GOT1/inhibitor has never been reported. This stimulated us to obtain the complex structure of GOT1 and $\mathrm{AH}$, in an attempt to accurately elucidate the specific mechanism of action between GOT1 and GOT1 inhibitors. After many attempts, the co- 
crystal structure of GOT1-AH was finally obtained, and crystallization of GOT1 in the presence of $\mathrm{AH}$ afforded the opportunity to gain more detailed insight into the binding mode of compound AH within the GOT1 active site. The resolution of the complex structure was obtained at 2.6 $\AA$ (PDB ID 6LIG). The statistics of data collection and refinement are listed in Table S1. The overall structure contains one large (residues 68-300) and two small (residues 17-67, 301-411) domains, with a core architecture consisting of an extended mixture of $\alpha$-helices and $\beta$-sheets through the active cavity of the protease, and has been previously described in detail (Figure 3A) [16,24]. The large regions in the dimer are fundamentally identical, while two active sites show different substrate accessibility [25]. We also tried to obtain the GOT1 complex with compounds 5 and 12, but failed to do so.

In contrast to WT-GOT1 (PDB ID 6DND), which contains a PLP as a cofactor in each monomer, the crystals soaked showed that chain $\mathrm{A}$ bound $\mathrm{AH}$ at the cleft in the interface of the subunits, whereas a PLP was situated in chain B (Figures 3B,C and S2). As expected, AH adopts a compact conformation in the bulk solvent of GOT1, which fully occupies the active site. The surface electrostatic potential indicates that the active pockets are electropositive, with some hydrophilic patches for substrate recognition (Figure S2). Structural comparison using the PDBeFold and DALI servers revealed that there are few changes in chain A between GOT1-AH and WT-GOT1, with a Z-score of 24.1 and root-mean-square deviation (RMSD) of $0.32 \AA$ over 410 residues. Overlapping these two structures, it was found that AH competitively binds to the PLP cofactor site of GOT1 (Figure S2). The hydroxylbutyrolactone core of $\mathrm{AH}$ is located in the same position as the phenolic oxygen of PLP, forming a rich interaction with surrounding amino acids. Moreover, the main difference is that Tyr71 and Ser297 in the opposite monomer constitute the active cavity of AH, which may play a role in stabilizing AH.
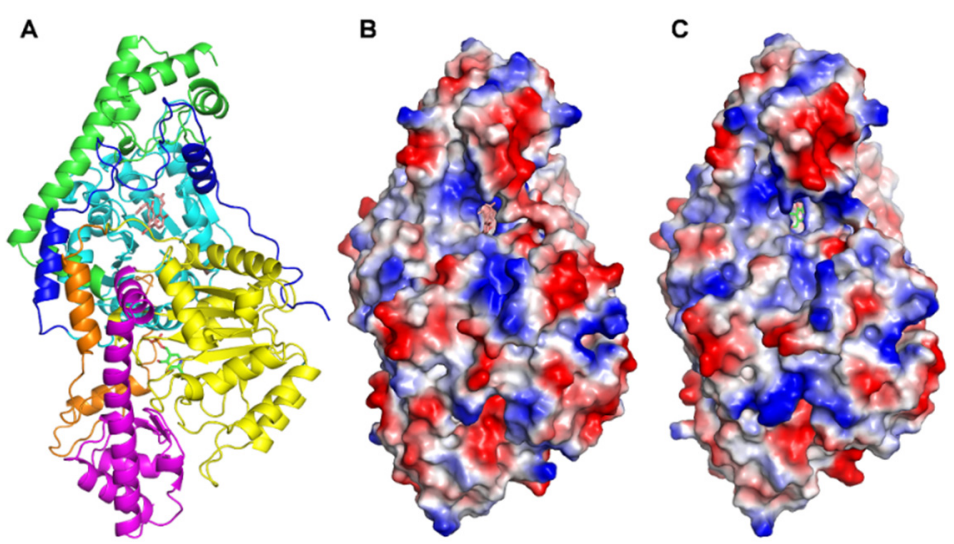

Figure 3. Structure of GOT1 in complex with AH: (A) Overall structure of GOT1-AH. The small domain 1 (blue/orange) includes two short $\alpha$-helix structures; domain 2 (cyan/yellow) is a smaller $(\alpha / \beta)_{7}$ sandwich fold; domain 3 (green/magenta) is close to domain 1. Surface representation of (B) AH and (C) PLP in the active site of GOT1. Electrostatic potential shows that the active pocket is positively charged. Blue: positive potential; red: negative potential; white: neutral potential.

Detailed analysis showed that AH is stacked in residues Gly39, Thr110, Trp141, Asp223, Tyr226, and Phe361 by hydrophobic interactions, while the isopentenyl group in $\mathrm{AH}$ at the entrance interacts with Ser297 and Tyr71 from the other subunit (Figure 4A). In addition, the side chains of Gly109, Ser258, Arg267, and Asn195 are hydrogen-bonded to $\mathrm{O} 4, \mathrm{O} 32$, and $\mathrm{O} 26$ of $\mathrm{AH}$, respectively (Figure 4B). Interestingly, the $\mathrm{C} 17$ atom of $\mathrm{AH}$ is covalently linked to the $\mathrm{N}$ atom of Lys259 in the large domain via an isopentyl linkage, similar to the PLP in WT-GOT1, which is connected to form an internal Schiff base via the oxygen atom on the aldehyde group [26]. According to the chemical structure of AH, it may form a strong $\pi-\pi$ stacking effect with GOT1. In chain B, the residual electron density clearly reveals the presence of PLP, which forms hydrogen bonds with Thr110, Tyr226, Ser258, and Arg267 (Figure S3). The rich hydrogen-bonding network in the active site 
contributes to stabilizing the spatial structure of proteins and the performance of their physiological functions $[27,28]$. When the hydrogen-bonding network formed by PLP is disturbed, the cofactors cannot be activated, resulting in the reduction or ablation of GOT1 activity.

A

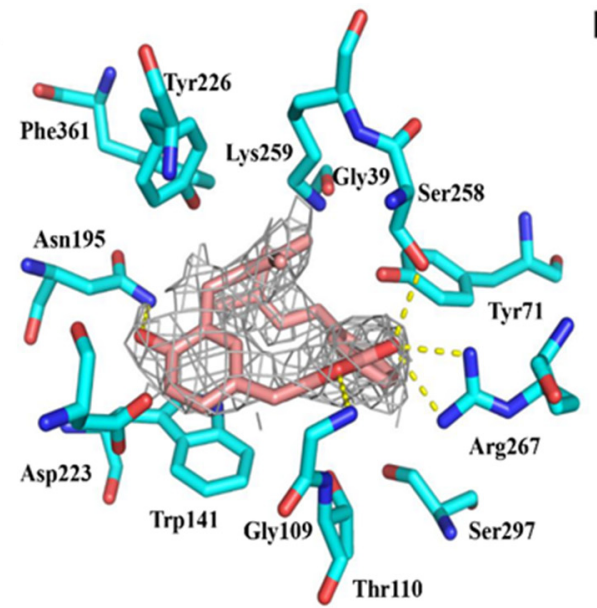

B

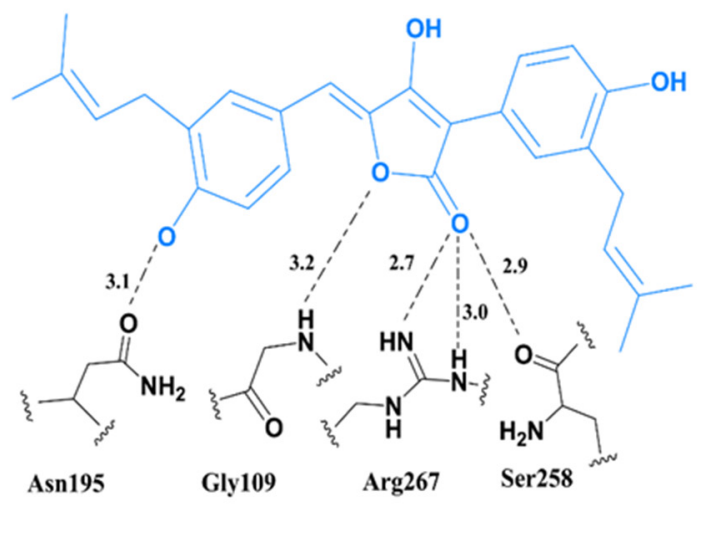

Figure 4. Detailed view of the active site: (A) The $2 F o-F c$ electron density of AH bound to chain A is shown as a gray mesh. The map is contoured at the $1 \sigma$ level. Side chains involved in the binding site are shown. (B) Schematic of the potential hydrogen-bonding network between AH (blue) and GOT1 (black).

\subsection{AH Inhibited Activity in Cancer Cells}

To explore the antitumor activity of $\mathrm{AH}$, several human cancer cell lines including pancreas (AsPC-1, PANC-1, SW1990), breast (HCC1806, MM231, MM453), colorectal (HCT116), and ovarian cancer cells (ES2), along with a nonmalignant human pancreatic duct epithelial cell line (HDPE6C7), were selected to detect the inhibitory effect of AH on their growth. After treatment for $48 \mathrm{~h}$, AH exhibited significant cytotoxicity effects on the SW1990, PANC-1, and AsPC-1 cell lines compared to the other cell lines, with an $\mathrm{IC}_{50}$ ranging from 6.32 to $10.47 \mu \mathrm{M}$ (Figure 5). Importantly, AH exhibited minimal cytotoxicity effects on the nonmalignant HPDE6C7 cell line, with an $\mathrm{IC}_{50}$ value over $100 \mu \mathrm{M}$. These data show that $\mathrm{AH}$ possesses selective anti-proliferation activity against PDAC cells.

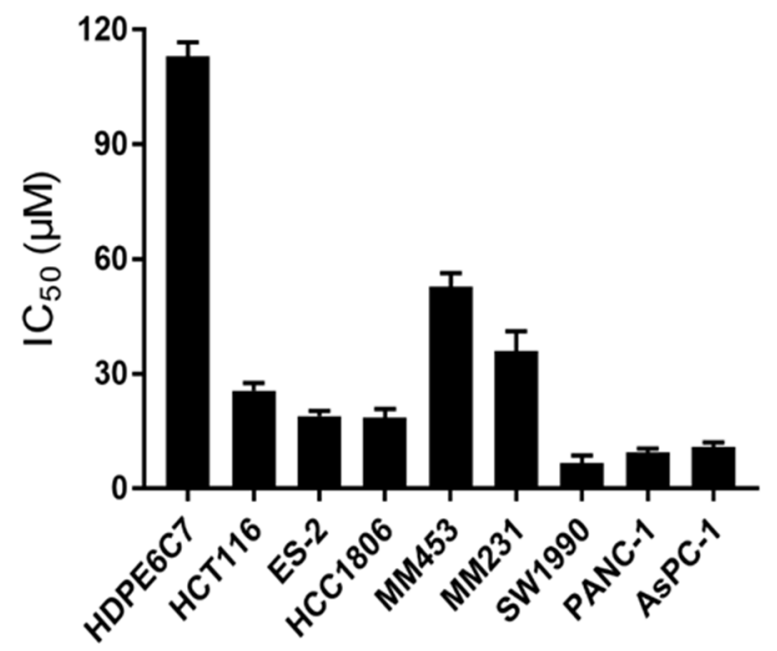

Figure 5. AH displays a higher growth inhibition rate in PDAC cells than in other cell lines. Pancreas cell lines (SW1990, AsPC-1, PANC-1), breast cell lines (HCC1806, MM231, MM453), HCT116, ES2, and a nonmalignant human pancreatic duct epithelial cell line (HDPE6C7) were treated with different concentrations of AH for $48 \mathrm{~h}$, and the MTT assay was used to measure cell viability. IC50 values were interpolated from the resulting dose-dependent curves. 


\subsection{AH Modulates Metabolism and ROS Response}

GOT1 is a key enzyme that is involved in the production of Gln-dependent NADPH in PDAC, and catalyzes the reversible conversion of Asp to OAA. To investigate the effects of $\mathrm{AH}$ on Gln metabolism, the associated metabolites including OAA and malate (Mal) were detected. As shown in Figure 6A, AH treatment reduced the content of OAA and Mal, while increasing Asp correspondingly. Consequently, the ratio of NADPH/NADP ${ }^{+}$ was remarkably decreased (Figure 6B). As excessive reactive oxygen species (ROS) can enhance cellular oxidative stress-resulting in damage to DNA, proteins, or lipids, causing apoptosis or necrosis [29] — we examined the physiological correlation between AH treatment and ROS response. 2,7-Dichlorofluorescein diacetate (DCFDA) was used to measure the intracellular ROS levels. In SW1990 cells, the concentration of ROS was positively correlated with the consumption of AH, indicating that cells inhibit the activity of GOT1 by upregulating ROS levels (Figure $6 \mathrm{C}, \mathrm{D}$ ). The above data indicate that $\mathrm{AH}$ treatment could block the production of Gln-dependent NADPH and participate in the redox homeostasis of PDAC cells.

\section{A}

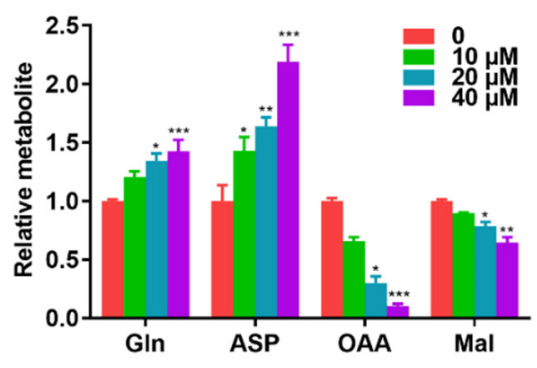

C

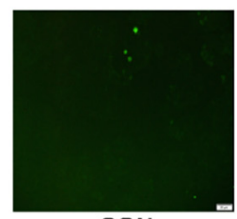

CON

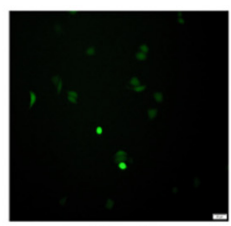

$20 \mu \mathrm{M}$

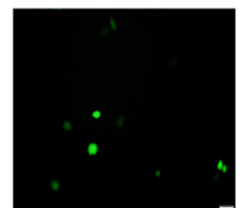

$10 \mu \mathrm{M}$

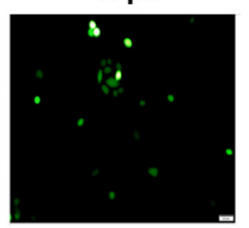

$40 \mu \mathrm{M}$
B

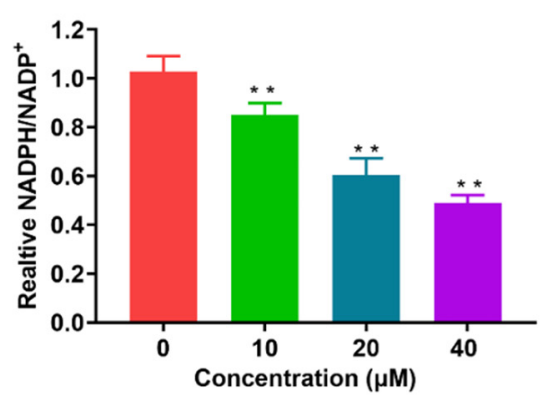

D

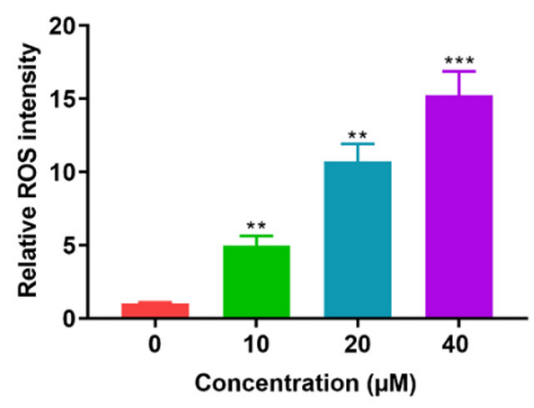

Figure 6. AH treatment induced Gln metabolism and maintained redox balance: (A) The effect of AH treatment $(10,20$, or $40 \mu \mathrm{M})$ on relative metabolite abundance in SW1990 cells. (B) The effect of AH treatment $(10,20$, or $40 \mu \mathrm{M})$ on the $\mathrm{NADP}^{+} / \mathrm{NADPH}$ ratio in SW1990 cells. (C,D) Intracellular ROS level measurement using carboxyH2DCFDA with 10,20 , or $40 \mu \mathrm{M}$ of AH. Data represent means $\pm \mathrm{SD}$ of three experiments. ${ }^{*} p<0.05,{ }^{* *} p<0.01,{ }^{* * *} p<0.001$ compared with the control group.

\subsection{AH Treatment Affected the Apoptosis, Cell Cycle, Proliferation, and Migration in} SW1990 Cells

In addition to inhibiting cell proliferation, we also investigated the mechanism of growth inhibition induced by $\mathrm{AH}$, via flow cytometry. The double staining of FITC-Annexin V and propidium iodide (PI) revealed that AH could induce apoptosis in SW1990 cells in a dosedependent manner. After $48 \mathrm{~h}$ incubation at concentrations of $0 \mu \mathrm{M}, 10 \mu \mathrm{M}, 20 \mu \mathrm{M}$, and $40 \mu \mathrm{M}$, the induction rates were $4.6 \%, 9.5 \%, 28.3 \%$, and $80.2 \%$, respectively (Figure $7 \mathrm{~A}$ ). Simultaneously, the cell cycle distributions of SW1990 cells were examined in the presence of increasing doses of AH treatment. As shown in Figure 7B, AH increased the cell number at the $\mathrm{S}$ phase in a dose-dependent manner, and led to a corresponding decrease in the G0/G1 phase. Meanwhile, there was only a slight increase in the G2/M phase when 
compared with high-dose AH treatment. In detail, AH could increase the proportion of S-phase cells $(25.1 \%, 32.5 \%, 38.6 \%$, and $43.9 \%$, respectively), with a decrease in the G0/G1 phase $(68.2 \%, 59.4 \%, 48.6 \%$, and $43.5 \%$, respectively) (Figure 7B). Subsequently, the EDU staining analysis and wound-healing cell migration test also proved the anti-proliferative effect of AH in SW1990 cells (Figure 7C-E), in a dose- and time-dependent manner. All of these data demonstrate that the S-phase arrest may be partially attributable to the reduction in the viability of PDAC cells by AH.
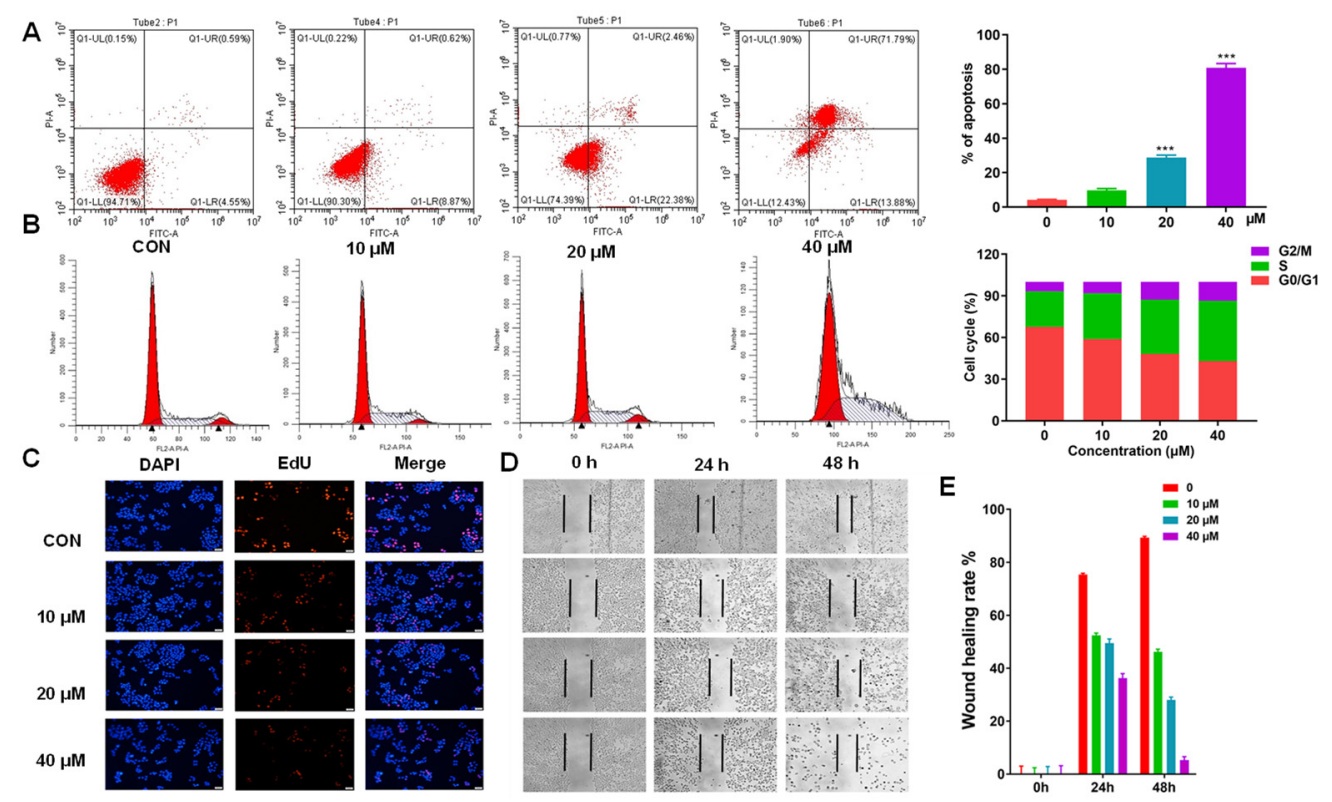

Figure 7. AH treatment affected the apoptosis, cell cycle, and proliferation in SW1990 cells: (A) Analysis of apoptosis in SW1990 cells using PI/Annexin V-FITC double staining after treatment with AH for $24 \mathrm{~h}$. (B) Cell cycle distribution analysis of SW1990 cells upon treatment with AH. (C) DAPI and EDU double staining was used to assay the effect of AH on the proliferation of SW1990 cells. (D) Wound-healing assay in SW1990 cells with indicated AH treatments at $0 \mathrm{~h}, 24 \mathrm{~h}$, and $48 \mathrm{~h}$. (E) Quantitative analysis of wound-healing assay. Data represent means \pm SD of three experiments. *** $p<0.001$ compared with the control group.

\subsection{In Vivo Antitumor Activity of $A H$}

Next, the antitumor effect of AH in vivo was evaluated in a CB-17/SCID murine xenograft model. One week after subcutaneous injection of PDAC cells into their lower flanks, the mice were subjected to intraperitoneal injection of vehicle or $\mathrm{AH}(2.5 \mathrm{mg} / \mathrm{kg} / \mathrm{d}$ and $5 \mathrm{mg} / \mathrm{kg} / \mathrm{d}$ ) for 14 days. As presented in Figure 8A-D, the tumor volume of the experimental group was significantly smaller than that of the control group, suggesting that AH had an inhibitory effect on the growth of SW1990 xenografts. Detection of tumor tissue metabolites found that OAA and Mal decreased, while Asp and Gln increased correspondingly. Therefore, the proportion of NADPH/NADP ${ }^{+}$was accompanied by a decrease, consistent with the effect in vitro (Figure $8 \mathrm{E}, \mathrm{F}$ ). Moreover, there were no significant changes in the body weight or histomorphology of the mice, indicating that $\mathrm{AH}$ is an effective and safe antitumor agent. 
A

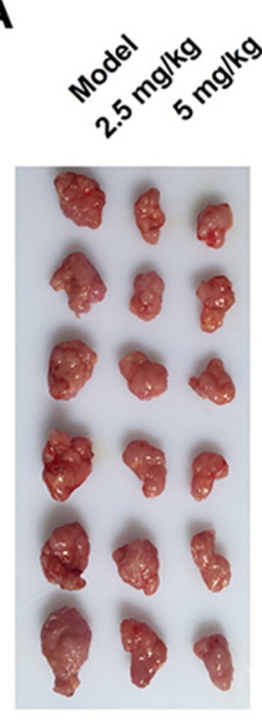

B
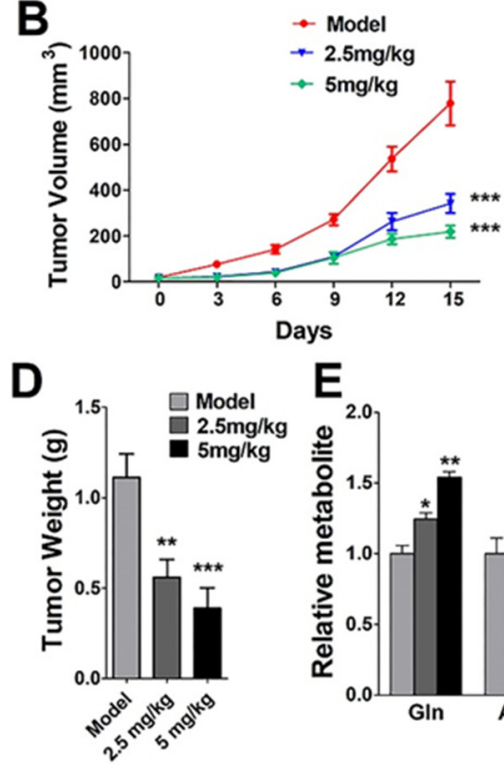
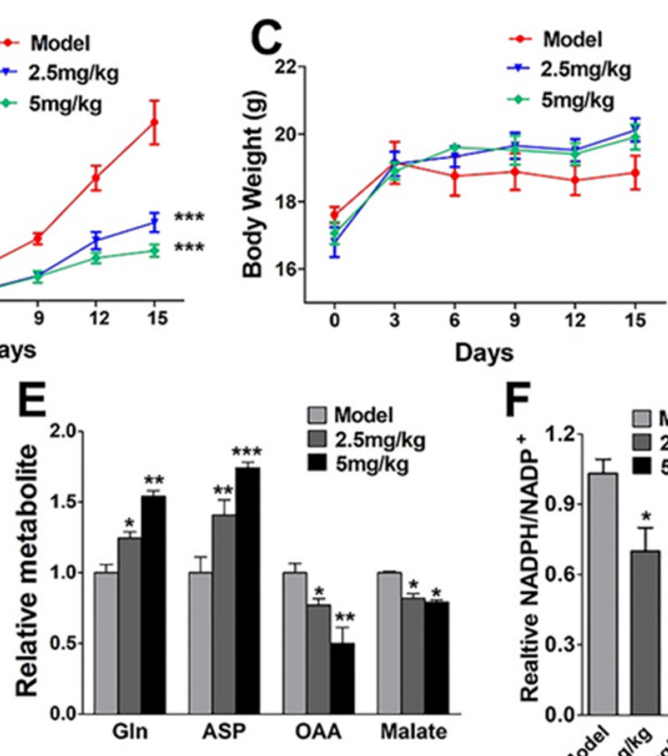

$\mathbf{F}$

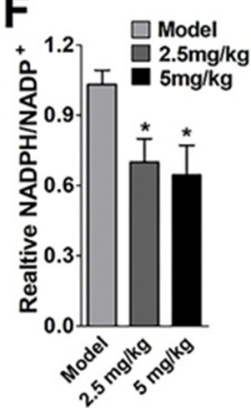

Figure 8. Evaluation of the in vivo efficacy of AH in xenograft tumors: (A) Tumor sample of xenograft mice after 14 days of treatment. (B) Tumor volume, (C) body weight, and (D) tumor weight for SW1990 xenografts in CB-17/SCID mice treated with 2.5 or $5 \mathrm{mg} / \mathrm{kg} /$ day of $\mathrm{AH}$ for 14 consecutive days. (E) Effects of metabolite abundance in different groups of tumor tissues. (F) NADPH/NADP+ ratio in different groups of tumor tissues. Analysis of variance (ANOVA) was used to compare the differences between groups. Data represent mean $\pm \mathrm{SD} .{ }^{*} p<0.05,{ }^{* *} p<0.01,{ }^{* * *} p<0.001$ compared with the control group.

\section{Materials and Methods}

\subsection{Fungal Material}

The marine sediment was collected in the Sanya Bay of the South China Sea, Sanya, China, in July 2019 (summer). The strain was collected from the marine sediment in August 2019 (summer). According to the morphology of this fungus, combined with the sequence analysis of the ITS region, the strain was identified as an Aspergillus terreus (GenBank accession number: OK465110). This A. terreus was preserved in Tongji Medical College, Huazhong University of Science and Technology.

\subsection{General Experimental Procedures, Fermentation, Extraction, and Isolation}

The high-resolution electrospray ionization mass spectra (HRESIMS) were recorded in positive-ion mode on a Thermo Fisher LC-LTQ-Orbitrap XL instrument. ECD data were measured with a JASCO-810 CD spectrometer instrument. Optical rotations UV data, and IR data were recorded on a PerkinElmer 341 instrument, a Varian Cary 50 instrument, and a Bruker Vertex 70 instrument with $\mathrm{KBr}$ pellets, respectively. Semi-preparative HPLC was conducted on a Dionex HPLC system equipped with an Ultimate 3000 pump (Thermo Fisher, Scientific, Waltham, MA, USA), an Ultimate 3000 autosampler injector, and an Ultimate 3000 diode array detector (DAD) controlled by Chromeleon software (version 6.80), using a reversed-phased C18 column $(5 \mu \mathrm{m}, 10 \times 250 \mathrm{~mm}$, Welch Ultimate XB-C18). Oneand two-dimensional NMR data were recorded on a Bruker AM-400 instrument, with the reference of ${ }^{1} \mathrm{H}$ and ${ }^{13} \mathrm{C}$ NMR chemical shifts of the solvent peaks for methanol- $\mathrm{d}_{4}$ $\left(\delta_{\mathrm{H}} 3.31\right.$ and $\left.\delta_{\mathrm{C}} 49.0\right)$ and $\mathrm{CHCl}_{3}-\mathrm{d}\left(\delta_{\mathrm{H}} 7.26\right.$ and $\left.\delta_{\mathrm{C}} 77.0\right)$. Silica gel $60 \mathrm{~F}_{254}$ was used for TLC (thin-layer chromatography) detection, and spots were visualized by spraying heated silica gel plates with $5 \% \mathrm{H}_{2} \mathrm{SO}_{4}$ in EtOH. Column chromatography (CC) was carried out using silica gel (80-120, 100-200, and 200-300 mesh, Qingdao Marine Chemical, Inc., Qingdao, China), LiChroprep RP-C 18 gel (40-63 $\mu \mathrm{m}$, Merck, Darmstadt, Germany), and Sephadex LH-20 (GE Healthcare Bio-Sciences AB, Bjorkgatan, Sweden). Experimental stains were incubated in potato dextrose agar (PDA) medium at $28^{\circ} \mathrm{C}$ for 7 days to prepare the seed cultures, which were then transferred into $1000 \mathrm{~mL}$ Erlenmeyer flasks, each containing 
$400 \mathrm{~g}$ of rice (total $100 \mathrm{~kg}$ ). After cultivation for 28 days, the medium was extracted with 95\% aqueous EtOH five times at room temperature. Afterwards, the solvent was removed under reduced pressure to yield a total residue, which was then suspended in water and partitioned repeatedly with EtOAc. The EtOAc extract $(1.2 \mathrm{~kg})$ was chromatographed on silica gel CC (80-120 mesh) using an increasing gradient of petroleum ether-ethyl acetate (100:0 to 0:100) to produce seven fractions (A-G).

Fraction D (112.3 g) was fractioned on silica gel CC (200-300 mesh) using an increasing gradient of petroleum ether-ethyl acetate (50:1 to 5:1) to produce eight subfractions (D1-D8). Subfraction D3 (12 g) was chromatographed on Sephadex LH-20 eluted with $\mathrm{CH}_{2} \mathrm{Cl}_{2}-\mathrm{MeOH}(1: 1, v / v)$ to yield four fractions (D3.1-D3.4). Fraction D3.2 (820 mg) was purified using semi-preparative HPLC eluted with $\mathrm{MeCN}-\mathrm{H}_{2} \mathrm{O}(63: 37, v / v, 2.0 \mathrm{~mL} / \mathrm{min}$ ), producing compounds $\mathbf{1}(112 \mathrm{mg}), \mathbf{2}(23 \mathrm{mg}), \mathbf{3}(41 \mathrm{mg})$, and $\mathbf{8}(7 \mathrm{mg})$. Fraction D3.3 was purified using semi-preparative HPLC $\left(\mathrm{MeCN}-\mathrm{H}_{2} \mathrm{O}, 57: 43, v / v, 2.0 \mathrm{~mL} / \mathrm{min}\right)$ to yield compounds $5(11 \mathrm{mg}), 7(16 \mathrm{mg})$, and $9(22 \mathrm{mg})$. Subfraction $\mathrm{D} 4(9 \mathrm{~g})$ was chromatographed on Sephadex LH-20 eluted with $\mathrm{CH}_{2} \mathrm{Cl}_{2}-\mathrm{MeOH}(1: 1, v / v)$ to yield three fractions (D4.1-D4.3). Compounds $\mathbf{6}(126 \mathrm{mg}), \mathbf{1 0}(5 \mathrm{mg}), \mathbf{1 1}(2 \mathrm{mg})$, and $\mathbf{1 7}(32 \mathrm{mg})$ were purified using semipreparative HPLC $\left(\mathrm{MeCN}-\mathrm{H}_{2} \mathrm{O}, 50: 50, v / v, 2.0 \mathrm{~mL} / \mathrm{min}\right)$ from fraction D4.1. Fraction D4.2 was purified using semi-preparative $\mathrm{HPLC}\left(\mathrm{MeOH}-\mathrm{H}_{2} \mathrm{O}, 70: 30, v / v, 2.0 \mathrm{~mL} / \mathrm{min}\right)$ to yield compounds $4(9 \mathrm{mg}), \mathbf{1 2}(7 \mathrm{mg})$, and $\mathbf{1 3}(2 \mathrm{mg})$. Fraction $\mathrm{E}(32 \mathrm{~g})$ was fractioned on silica gel CC (200-300 mesh) using an increasing gradient of petroleum ether-ethyl acetate (50:1 to 1:1) to produce four subfractions (E1-E4). Subfraction E3 (4 g) was chromatographed using an $\mathrm{RP}^{-} \mathrm{C}_{18}$ column with $\mathrm{MeOH}-\mathrm{H}_{2} \mathrm{O}$ (from 30:70 to 90:10, v/v) to produce four subfractions (E3.1-E3.4). Compounds $\mathbf{1 4}(11 \mathrm{mg}), \mathbf{1 3}(5 \mathrm{mg}), \mathbf{1 5}(7 \mathrm{mg})$, and $\mathbf{1 8}(8 \mathrm{mg})$ were purified from

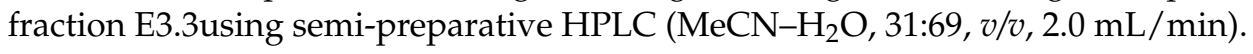

\subsection{Materials}

Antibodies against GOT1 and $\beta$-actin were purchased commercially from Santa Cruz Biotechnology; goat anti-mouse IgG and goat anti-rabbit IgG antibodies were purchased from Cell Signaling Technology. Benzyloxycarbonyl-Val-Ala-Asp fluoromethylketone (ZVAD-FMK) was obtained from Selleck; 20,70-dichlorodihydrofluorescein diacetate (DCFHDA) was obtained from Invitrogen.

\subsection{Molecular Cloning, Expression, and Purification}

The expression construct pET26b-got1 (Invitrogen, Carlsbad, NJ, USA) was C-terminal His $_{6}$-tagged and transformed into E. coli BL21 (DE3) cells at $30{ }^{\circ} \mathrm{C}$ for $6 \mathrm{~h}$. The recombinant protein was initially passed over a HisTrap ${ }^{\mathrm{TM}}$ FF crude column (GE Healthcare, $5 \mathrm{~mL}$, Pittsburgh, PA, USA). Peak elution fractions containing GOT1 were collected and concentrated using centrifuge tubes (Millipore, Billerica, MA, USA) with a $10 \mathrm{kDa}$ cutoff, and then purified via anion-exchange chromatography with Resource Q (GE Healthcare, $5 \mathrm{~mL}$, Pittsburgh, PA, USA). Subsequently, the sample was injected onto a Superdex200 Increase 10/300 GL Column (GE Healthcare, Pittsburgh, PA, USA) and dialyzed in $20 \mathrm{mM}$ HEPES buffer ( $\mathrm{pH} 7.5$ ) and $200 \mathrm{mM} \mathrm{NaCl}$.

\subsection{Enzyme Inhibition Assays}

The GOT1 enzymatic activity assay was carried out using a 96-well plate reader as previously reported [10]. The $100 \mu \mathrm{L}$ reaction system contained $0.1 \mathrm{mg} / \mathrm{mL}$ recombinant GOT1 protein, $1 \mathrm{mM} \alpha-\mathrm{KG}, 1 \mathrm{mM} \mathrm{NADH}, 4 \mathrm{mM}$ Asp, and $1 \mathrm{U} / \mathrm{mL}$ malate dehydrogenase, and measured absorbance at a wavelength of $340 \mathrm{~nm}$. The degree of absorbance decrease was in direct proportion to GOT1 activity. The inhibitory activity in vitro was measured by adding different concentrations of compounds. SigmaPlot with the PlotEnzyme Kinetics Module was used to analyze the enzyme activity. 


\subsection{Microscale Thermophoresis}

The Monolith NTTM Protein Labeling Kit was used to test the binding capacity of proteins to AH, according to the manufacturer's instructions. GOT1 was labeled and diluted in a buffer containing $20 \mathrm{mM}$ HEPES (pH 7.5) and Tween-20 $(0.5(v / v) \%)$, and then incubated with different concentration of $\mathrm{AH}$ for $10 \mathrm{~min}$ at $37^{\circ} \mathrm{C}$. Subsequently, the materials were put into standard capillary tubes and tested for thermophoresis using a Monolith NT.115 instrument (NanoTemper Technologies, München, Germany). The parameters were set to temperature $25^{\circ} \mathrm{C}$, LED power $100 \%$, and laser power $40 \%$, with a $30 \mathrm{~s}$ on-time. NTAnalysis software was used to calculate the $K_{d}$ values.

\subsection{Protein Crystallization}

Protein crystals of GOT1 were grown via the sitting-drop vapor diffusion method after $48 \mathrm{~h}$ at $18{ }^{\circ} \mathrm{C}$, with $0.25 \mu \mathrm{L}$ of protein and $0.25 \mu \mathrm{L}$ of precipitant solution consisting of $0.1 \mathrm{M}$ HEPES pH 7.5, 0.005 M nickel(II) chloride hexahydrate, $0.005 \mathrm{M}$ cobalt(II) chloride hexahydrate, $0.005 \mathrm{M}$ magnesium chloride hexahydrate, $0.005 \mathrm{M}$ cadmium chloride hydrate, and $12 \% w / v$ polyethylene glycol 3350 . Before crystallization, WT-GOT1 $(16 \mathrm{mg} / \mathrm{mL})$ was mixed with $\mathrm{AH}$ at a final concentration of $40 \mu \mathrm{M}$ and incubated on ice for $2 \mathrm{~h}$, and then centrifuged to remove the precipitate. Formed crystals were transferred in mother liquor supplemented with $25 \%$ glycerol and flash-cooled in liquid nitrogen. Diffraction data of GOT1 were collected using a beamline BL-19U at the Shanghai Synchrotron Radiation Facility in China, and processed using the HKL-2000 package [22]. The structure of the complex was determined via molecular replacement using the human GOT1 (PDB entry 6DND) with the Phaser crystallographic software in a stepwise manner [16,30]. Subsequently, PHENIX and COOT software were used to manually refine and adjust the structural models [26,31]. PyMOL software was used to create the figures.

\subsection{Cell Culture and Viability Assay}

Pancreas (SW1990, AsPC-1, PANC-1), colorectal (HCT116), breast (HCC1806, MM231, MM453), and ovarian cancer cells (ES2), along with a nonmalignant human pancreatic duct epithelial cell line (HDPE6C7), were obtained from ATCC, and were cultured in 1640 medium or Dulbecco's modified Eagle medium (DMEM) supplemented with $10 \%(v / v)$ fetal bovine serum (FBS), $100 \mu \mathrm{g} / \mathrm{mL}$ streptomycin, and $100 \mathrm{U} / \mathrm{mL}$ penicillin. Cells were maintained in a humidified atmosphere of $5 \% \mathrm{CO}_{2}$ at $37{ }^{\circ} \mathrm{C}$. MTT (3- $(4,5-$ dimethylthiazol-2-yl)-2,5-diphenyl tetrazolium bromide) assay was used to assess the cell viability. Briefly, cells were seeded in 96-well plates at a density of $5 \times 10^{3}$ cells per well for $24 \mathrm{~h}$. Then, the medium was removed, and the cells were treated with different concentrations $(0.1-100 \mu \mathrm{M})$ of $\mathrm{AH}$, with DMSO as a vehicle control. After incubation for $48 \mathrm{~h}, 100 \mu \mathrm{L}$ of MTT solution $(2 \mathrm{mg} / \mathrm{mL})$ was added to each well, and the wells were incubated at $37^{\circ} \mathrm{C}$ for another $4 \mathrm{~h}$. The formed formazan crystals were dissolved in DMSO $(200 \mu \mathrm{L} /$ well $)$ and shaken for $5 \mathrm{~min}$. Then, the absorbance of the solution was measured at $490 \mathrm{~nm}$ with a microplate reader. $\mathrm{IC}_{50}$ values were interpolated from the resulting dose-dependent curves using GraphPad Prism 7.0. The reported $\mathrm{IC}_{50}$ values are the average of three independent experiments, each consisting of six replicates per concentration level (overall $n=18$ ).

\subsection{Cell Proliferation Assay}

Cells were seeded in 96-well plates at a density of $2 \times 10^{3}$ cells per well. To remove OAA, cells were inoculated in complete medium ( $2 \mathrm{mM} \mathrm{Gln}$ and $10 \mathrm{mM} \mathrm{Glu})$, which was then replaced the same day with culture medium supplemented with $10 \%$ FBS. The cells were stained with $0.1 \%$ crystal violet after being fixed in $10 \%$ formalin. After extracting the dye with $10 \%$ acetic acid, relative proliferation was determined by measuring the OD at $595 \mathrm{~nm}$. 


\subsection{Cell Cycle Assay}

The phase distribution of DNA content was determined via propidium iodide (PI) staining. Cells were treated with varying doses of $\mathrm{AH}$ or vehicle for $48 \mathrm{~h}$, and then the cell pellets were stained in the dark for $30 \mathrm{~min}$ with $60 \mu \mathrm{g} / \mathrm{mL}$ RNase A and $30 \mu \mathrm{g} / \mathrm{mL}$ PI. Finally, CellQuest software (Becton-Dickinson, Franklin Lakes, NJ, USA) was used to perform flow cytometric studies. Three separate experiments were used to determine the cell cycle.

\subsection{Cell Apoptosis Assay}

The Annexin V-FITC apoptosis kit was purchased from USA BD PharMingen ${ }^{\mathrm{TM}}$. In brief, different concentrations of $\mathrm{AH}$ were added to the wells for $48 \mathrm{~h}$, and then the cells were harvested, before being resuspended in Annexin-binding buffer and incubated with Annexin V-FITC $(10 \mathrm{mg} / \mathrm{mL})$ and PI in the dark for $15 \mathrm{~min}$. Flow cytometry was used to detect the stained cells and analyze the apoptosis immediately.

\subsection{Wound-Healing Assay}

SW1990 cells were seeded in 6-well plates for $24 \mathrm{~h}$. Each well was scratched with a $10 \mu \mathrm{L}$ pipette tip, and then the plate was washed with PBS to peel off the suspended cells, before adding different concentrations of $\mathrm{AH}(0-40 \mu \mathrm{M})$. Images from the same area of the wound were taken separately under a microscope from 0 to $48 \mathrm{~h}$.

\subsection{Metabolomics Analysis}

SW1990 cells were cultivated to around 50\% confluence in DMEM media with $10 \mathrm{mM}$ glucose, $2 \mathrm{mM}$ glutamine, and 5\% FBS. A complete medium was prepared prior to two hours of metabolite collection. Quantification kits were used to determine the abundance of Asp (Abcam, \#ab102512, Cambridge, UK), Gln (BioVision, \#K55, San Francisco, CA, USA), malate (BioVision, \#K637, San Francisco, CA, USA), and OAA (Abcam, \#ab83428, Cambridge, UK). According to the manufacturer's instructions, we collected some cells and homogenized them in the buffers provided. The supernatants were centrifuged, and then deproteinized using a $10 \mathrm{~K}$ spin column before being analyzed and compared to standard curves.

\subsection{Xenograft Studies}

Animal treatment and experiments were conducted in the Experimental Animal Center. Commercial 8-week-old CB-17/SCID mice (male) were purchased from Huafukang, China. After being resuspended using $0.1 \mathrm{~mL}$ of PBS, SW1990 cells $\left(3 \times 10^{6}\right)$ were injected subcutaneously into the lower flanks of the mice. When the tumors attained a diameter of $\sim 3 \mathrm{~mm}$, the mice were randomly divided into three groups- $2.5 \mathrm{mg} / \mathrm{kg}, 5 \mathrm{mg} / \mathrm{kg}$, and control-and were subsequently injected intraperitoneally with normal saline or $\mathrm{AH}$ every day. A Vernier caliper was used to measure the size of the tumors. After two weeks of therapy, mice were euthanized under deep anesthesia (chloral hydrate, $10 \%$, $10 \mu \mathrm{L} / \mathrm{g}$ ), and tumor tissue metabolites were detected using the above method. All animal studies were carried out in accordance with the Huazhong University of Science and Technology's guidelines.

\subsection{Statistical Analysis}

The collected data were presented as mean \pm SD. The statistical analysis was performed using the GraphPad Prism 7.0 program. ANOVA (one-way analysis of variance) used SPSS version 13.0 software to evaluate the multiple-group comparisons, with values of $p<0.05$ deemed statistically significant.

\section{Conclusions}

In this study, 18 butyrolactone derivatives (1-18) were obtained from the solid culture extract of a marine-derived Aspergillus terreus. Among those isolates, aspulvinone $\mathrm{H}$ 
$(\mathrm{AH}, 6)$ was found to bear remarkable GOT1 inhibitory activity in vitro, with an $\mathrm{IC}_{50}$ value of $(5.91 \pm 0.04) \mu \mathrm{M}$. Importantly, the crystal structure of GOT1-AH suggests that AH and the cofactor pyrido-aldehyde 5-phosphate competitively bind to the active sites of GOT1, likely representing the molecular mechanism of action between AH and GOT1. More notably, the crystal structure of GOT1-AH is the first reported complex structure of GOT1/inhibitor. Furthermore, in vitro biological studies indicated that AH can suppress glutamine metabolism, making PDAC cells sensitive to oxidative stress, and inhibiting cell proliferation; this metabolite also exhibited potent in vivo antitumor activity in an SW1990-cell-induced xenograft model. In our previous paper, we identified aspulvinone $\mathrm{O}$ (AO) as a potent butyrolactone-type GOT1 inhibitor from soil-sourced A. terreus collected from the bottom of the Yangzi River. AH featured a similar chemical structure and activity to those of $\mathrm{AO}$, and both compounds possessed outstanding GOT1-inhibitory activity, and could suppress PADC cells' growth by interfering with glutamine metabolism, illustrating the significant role of 5-benzylidene-3-phenylfuran-2 $(5 H)$-one-containing butyrolactones in the development of natural GOT1 inhibitors. In conclusion, the discovery of AH not only provides a promising lead compound for the development of novel GOT1-inhibitory agents, but also represents an attractive potential molecule for anti-PDAC drug development.

Supplementary Materials: The following are available online at https://www.mdpi.com/article/10 .3390/md19110588/s1, Figures S1-S39, Table S1: Data collection and refinement statistics.

Author Contributions: Conceptualization, W.S. (Wei Song) and Q.X.; data curation, S.Y.; funding acquisition, C.Q. and Y.Z.; investigation, C.Q. and Y.Z.; methodology, C.Q., W.S. (Weiguang Sun) and Y.Z.; project administration, Y.Z. and L.G.; software, S.Y.; supervision, W.S. (Weiguang Sun) and Y.Z.; validation, S.Y. and W.S. (Weiguang Sun); writing-original draft, S.Y. and C.Q.; writing-review and editing, S.Y., C.Q., W.S. (Weiguang Sun) and Y.Z. All authors have read and agreed to the published version of the manuscript.

Funding: This research was funded by the program for Changjiang Scholars of the Ministry of Education of the People's Republic of China, grant number T2016088; the National Natural Science Foundation for Distinguished Young Scholars of China, grant number 81725021; the Innovative Research Groups of the National Natural Science Foundation of China, grant number 81721005; the National Natural Science Foundation of China (No. 82003633); and the Open Foundation of Hubei Key Laboratory of Wudang Local Chinese Medicine Research (WDCM2019004).

Institutional Review Board Statement: All experiments were carried out according to the NIH Guidelines for Care and Use of Laboratory Animals and approved by the Bioethics Committee of Shanghai Institute of Materia Medica and the Animal Care and Use Committee of Tongji Medical College, with the protocol number of ACUC-TMC 2020-Ani2113.

Data Availability Statement: Not applicable.

Acknowledgments: We thank the Analytical and Testing Center at Huazhong University of Science and Technology for assistance in the performance of ECD, UV, and IR analyses. This work was supported by the program for the Academic Frontier Youth Team of HUST and the Integrated Innovative Team for Major Human Diseases Program of Tongji Medical College (HUST).

Conflicts of Interest: The authors declare no conflict of interest.

\section{References}

1. Vincent, A.; Herman, J.; Schulick, R.; Hruban, R.H.; Goggins, M. Pancreatic cancer. Lancet 2011, 378, 607-620. [CrossRef]

2. Collisson, E.A.; Bailey, P.; Chang, D.K.; Biankin, A.V. Molecular subtypes of pancreatic cancer. Nat. Rev. Gastroenterol. Hepatol. 2019, 16, 207-220. [CrossRef]

3. Heiden, M.G.V.; Cantley, L.C.; Thompson, C.B. Understanding the warburg effect: The metabolic requirements of cell proliferation. Science 2009, 324, 1029-1033. [CrossRef] [PubMed]

4. Son, J.Y.; Lyssiotis, C.A.; Hao, Q.Y.; Wang, X.X.; Hua, S.J.; Ligorio, M.; Perera, R.M.; Ferrone, C.R.; Mullarky, E.; Chang, N.S.; et al. Glutamine supports pancreatic cancer growth through a KRAS-regulated metabolic pathway. Nature 2013, 496, 101-105. [CrossRef] [PubMed]

5. Li, D.D.; Fu, Z.Q.; Chen, R.W.; Zhao, X.H.; Zhou, Y.; Zeng, B.M.; Yu, M.; Zhou, Q.B.; Lin, Q.; Gao, W.C.; et al. Inhibition of glutamine metabolism counteracts pancreatic cancer stem cell features and sensitizes cells to radiotherapy. Oncotarget 2015, 6, 31151-31163. [CrossRef] [PubMed] 
6. Ma, X.; Moore, Z.R.; Huang, G.; Huang, X.; Boothman, D.A.; Gao, J. Nanotechnology-enabled delivery of NQO1 bioactivatable drugs. J. Drug Target 2015, 23, 672-680. [CrossRef] [PubMed]

7. Lyssiotis, C.A.; Son, J.; Cantley, L.C.; Kimmelman, A.C. Pancreatic cancers rely on a novel glutamine metabolism pathway to maintain redox balance. Cell Cycle 2013, 12, 1987-1988. [CrossRef]

8. Halbrook, C.J.; Lyssiotis, C.A. Employing metabolism to improve the diagnosis and treatment of pancreatic cancer. Cancer Cell 2017, 31, 5-19. [CrossRef]

9. Weinberg, F.; Hamanaka, R.; Wheaton, W.W.; Weinberg, S.; Joseph, J.; Lopez, M.; Kalyanaraman, B.; Mutlu, G.M.; Budinger, G.R.; Chandel, N.S. Mitochondrial metabolism and ROS generation are essential for Kras-mediated tumorigenicity. Proc. Natl. Acad. Sci. USA 2010, 107, 8788-8793. [CrossRef]

10. Sun, W.G.; Luan, S.S.; Qi, C.X.; Yan, S.; Li, H.; Zhang, Y.H. Aspulvinone O, a natural inhibitor of GOT1 suppresses pancreatic ductal adenocarcinoma cells growth by interfering glutamine metabolism. Cell Commun. Signal. 2019, 17, 111. [CrossRef]

11. Abrego, J.; Gunda, V.; Vernucci, E.; Shukla, S.K.; King, R.J.; Dasgupta, G.A.; Murthy, D.; Yu, F.; Singh, P.K. GOT1-mediated anaplerotic glutamine metabolism regulates chronic acidosis stress in pancreatic cancer cells. Cancer Lett. 2017, 400, 37-46. [CrossRef] [PubMed]

12. Wise, D.R.; Thompson, C.B. Glutamine addiction: A new therapeutic target in cancer. Trends Biochem. Sci. 2010, 35, 427-433. [CrossRef] [PubMed]

13. Obara-Michlewska, M.; Szeliga, M. Targeting glutamine addiction in gliomas. Cancers 2020, 12, 310. [CrossRef] [PubMed]

14. Holt, M.C.; Assar, Z.; Zavareh, R.B.; Lin, L.; Anglin, J.; Mashadova, O.; Haldar, D.; Mullarky, E.; Kremer, D.M.; Cantley, L.C.; et al. Biochemical characterization and structure-based mutational analysis provides insight into binding and mechanism of action of novel aspartate aminotransferase inhibitors. Biochemistry 2018, 57, 6604-6614. [CrossRef] [PubMed]

15. Śledź, P.; Caflisch, A. Protein structure-based drug design: From docking to molecular dynamics. Curr. Opin. Struct. Biol. 2017, 48, 93-102. [CrossRef] [PubMed]

16. Cruz, P.G.; Auld, D.S.; Schultz, P.J.; Lovell, S.; Battaile, K.P.; MacArthur, R.; Shen, M.; Castillo, G.T.; Inglese, J.; Sherman, D.H. Titration-based screening for evaluation of natural product extracts: Identification of an aspulvinone family of luciferase inhibitors. Chem. Biol. 2011, 18, 1442-1452. [CrossRef] [PubMed]

17. Niu, X.; Dahse, H.M.; Menzel, K.D.; Lozach, O.; Walther, G.; Meijer, L.; Grabley, S.; Sattler, I. Butyrolactone I derivatives from Aspergillus terreus carrying an unusual sulfate moiety. J. Nat. Prod. 2008, 71, 689-692. [CrossRef]

18. Min, B.S.; Na, M.K.; Oh, S.R.; Ahn, K.S.; Jeong, G.S.; Li, G.; Lee, S.K.; Joung, H.; Lee, H.K. New furofuran and butyrolactone lignans with antioxidant activity from the stem bark of Styrax japonica. J. Nat. Prod. 2004, 67, 1980-1984. [CrossRef]

19. Qi, C.; Gao, W.; Guan, D.; Wang, J.; Liu, M.; Chen, C.; Zhu, H.; Zhou, Y.; Lai, Y.; Hu, Z.; et al. Butenolides from a marine-derived fungus Aspergillus terreus with antitumor activities against pancreatic ductal adenocarcinoma cells. Bioorg. Med. Chem. 2018, 26, 5903-5910. [CrossRef]

20. Hamed, A.; Abdel-Razek, A.S.; Omran, D.A.; El-Metwally, M.M.; El-Hosari, D.G.; Frese, M.; Soliman, H.S.M.; Sewald, N.; Shaaban, M. Terretonin O: A new meroterpenoid from Aspergillus terreus. Nat. Prod. Res. 2020, 34, 965-974. [CrossRef]

21. Qi, C.; Gao, W.; Wang, J.; Liu, M.; Zhang, J.; Chen, C.; Hu, Z.; Xue, Y.; Li, D.; Zhang, Q.; et al. Terrusnolides A-D, new butenolides with anti-inflammatory activities from an endophytic Aspergillus from Tripterygium wilfordii. Fitoterapia 2018, 130, 134-139. [CrossRef]

22. Palmer, R.B. Gamma-butyrolactone and 1,4-butanediol: Abused analogues of gamma-hydroxybutyrate. Toxicol Rev. 2004, 23, 21-31. [CrossRef]

23. Liu, M.; Qi, C.; Sun, W.; Shen, L.; Wang, J.; Liu, J.; Lai, Y.; Xue, Y.; Hu, Z.; Zhang, Y. $\alpha$-Glucosidase inhibitors from the coral-associated fungus Aspergillus terreus. Front. Chem. 2018, 6, 422. [CrossRef]

24. Rhee, S.; Silva, M.M.; Hyde, C.C.; Rogers, P.H.; Metzler, C.M.; Metzler, D.E.; Arnone, A. Refinement and comparisons of the crystal structures of pig cytosolic aspartate aminotransferase and its complex with 2-methylaspartate. J. Biol. Chem. 1997, 272, 17293-17302. [CrossRef] [PubMed]

25. Dajnowicz, S.; Parks, J.M.; Hu, X.C.; Gesler, K.; Kovalevsky, A.Y.; Mueser, T.C. Direct evidence that an extended hydrogenbonding network influences activation of pyridoxal 5-phosphate in aspartate aminotransferase. J. Biol. Chem. 2017, 292, 5970-5980. [CrossRef] [PubMed]

26. Mccoy, A.J.; Grossekunstleve, R.W.; Adams, P.D.; Winn, M.D.; Storoni, L.C.; Read, R.J. Phaser crystallographic software. J. Appl. Crystallogr. 2007, 40, 658-674. [CrossRef] [PubMed]

27. Dajnowicz, S.; Johnston, R.C.; Parks, J.M.; Blakeley, M.P.; Keen, D.A.; Weiss, K.L.; Gerlits, O.; Kovalevsky, A.; Mueser, T.C. Direct visualization of critical hydrogen atoms in a pyridoxal 5'-phosphate enzyme. Nat. Commun. 2017, 8, 955. [CrossRef] [PubMed]

28. Bifulco, M.; Malfitano, A.M.; Proto, M.C.; Santoro, A.; Caruso, M.G.; Laezza, C. Biological and pharmacological roles of N6-isopentenyladenosine: An emerging anticancer drug. Anticancer Agents Med. Chem. 2008, 8, 200-204. [CrossRef] [PubMed]

29. Zhou, B.; Zhang, J.Y.; Liu, X.S.; Chen, H.Z.; Ai, Y.L.; Cheng, K.; Sun, R.Y.; Zhou, D.W.; Han, J.H.; Wu, Q. Tom20 senses iron-activated ROS signaling to promote melanoma cell pyroptosis. Cell Res. 2018, 28, 1171-1185. [CrossRef]

30. Terwilliger, T.C. Automated structure solution, density modification and model building. Acta Crystallogr. D Biol. Crystallogr. 2002, 58, 1937-1940. [CrossRef]

31. Emsley, P.; Cowtan, K. Coot: Model-building tools for molecular graphics. Acta Crystallogr. D Biol. Crystallogr. 2004, 60, 2126-2132. [CrossRef] [PubMed] 